\title{
Ultraviolet radiation-induced inflammation activates $\beta$-catenin signaling in mouse skin and skin tumors
}

\author{
RAM PRASAD ${ }^{1}$ and SANTOSH K. KATIYAR ${ }^{1,2}$ \\ ${ }^{1}$ Department of Dermatology, University of Alabama at Birmingham, Birmingham, AL 35294; \\ ${ }^{2}$ Birmingham Veterans Affairs Medical Center, Birmingham, AL 35233, USA
}

Received December 5, 2013; Accepted January 7, 2014

DOI: $10.3892 /$ ijo.2014.2275

\begin{abstract}
UVB-induced inflammation, in particular the overexpression of cyclooxygenase-2 (COX-2) and prostaglandin (PG) E2, has been implicated in photocarcinogenesis. UVB-induced COX-2 has been associated with $\beta$-catenin signaling in keratinocytes. However, a definitive role for COX- 2 in the activation of $\beta$-catenin signaling as well as its role in UVB-induced skin tumors has not been established. We report that exposure of the skin to UVB resulted in a timeand dose-dependent activation of $\beta$-catenin in $\mathrm{C} 3 \mathrm{H} / \mathrm{HeN}$ mice. This response was COX-2-dependent as UVB-exposed COX-2-deficient mice exhibited significantly lower levels of UVB-induced activation of $\beta$-catenin. Moreover, treatment of mice with indomethacin, a COX-2 inhibitor, and an EP2 antagonist inhibited UVB-induced $\beta$-catenin signaling. Exposure of SKH-1 hairless mice to UVB radiation $(180 \mathrm{~mJ} /$ $\left.\mathrm{cm}^{2}\right) 3$ times a week for 24 weeks resulted in activation of $\beta$-catenin signaling in UVB-irradiated skin as well as UVB-induced skin tumors. Concomitantly, the levels of CK $1 \alpha$ and GSK-3 $\beta$, which are responsible for $\beta$-catenin signaling, were reduced while the levels of $\mathrm{c}-\mathrm{Myc}$ and cyclin D1, which are downstream targets of $\beta$-catenin, were increased. To further verify the role of UVB-induced inflammation in activation of $\beta$-catenin signaling, a high-fat-diet model was used. Administration of high-fat diet exacerbated UVB-induced inflammation. Administration of the high-fat diet enhanced $\beta$-catenin signaling and the levels of its downstream targets (c-Myc, cyclin D1, cyclin D2, MMP-2 and MMP-9) in UVB-exposed skin and skin tumors in SKH-1 mice. These data suggest that UV-induced $\mathrm{COX}-2 / \mathrm{PGE}_{2}$ stimulates $\beta$-catenin signaling, and that $\beta$-catenin activation may contribute to skin carcinogenesis.
\end{abstract}

Correspondence to: Dr Santosh K. Katiyar, Department of Dermatology, University of Alabama at Birmingham, 1670, University Boulevard, Volker Hall 557, Birmingham, AL 35294, USA

E-mail: skatiyar@uab.edu

Key words: cyclooxygenase-2, prostaglandins, ultraviolet radiation, $\beta$-catenin, skin tumor, inflammation

\section{Introduction}

The incidence of skin cancers, including melanoma and nonmelanoma, is equivalent to the incidence of malignancies in all other organs combined (1). It is estimated that approximately 2 million people are diagnosed with squamous and basal cell skin cancers annually in the US (2). Epidemiological and clinical studies indicate that ultraviolet B (UVB) radiation acts as a tumor initiator, tumor promoter as well as a complete carcinogen for the development of skin neoplasms (3). Exposure of the skin to solar UV radiation induces inflammatory responses, oxidative stress, alterations in cell cycle progression, DNA damage and suppression of immune responses, all of which have been implicated in the development of skin cancers (3-7). $\mathrm{UV}$-induced inflammation is considered as an early event in skin tumor promotion and the growth of tumors, and chronic and sustained inflammation plays an important role in all three stages of tumor development, i.e., initiation, promotion and progression (3). The UV-induced inflammatory responses are characterized by the development of hyperplastic responses, and increases in the levels of cyclooxygenase-2 (COX-2) and prostaglandin (PG) metabolites (3). A link between $\mathrm{COX}-2 / \mathrm{PGE}_{2}$ and $\beta$-catenin signaling has been observed in colon cancer in which COX-2 is overexpressed and $\beta$-catenin signaling contributes to tumor cell growth (8). The $\beta$-catenin signaling pathway also has been implicated in the growth of melanomas as well as in the migration of melanoma cells (9-11).

$\beta$-catenin, a $90-\mathrm{kDa}$ cytosolic protein, is an important component of the Wnt pathway. Wnt/ $\beta$-catenin proteins regulate expression of various target genes that mediate cellular processes including proliferation and migration. Activation of $\mathrm{Wnt} / \beta$-catenin proteins and mutations in $\beta$-catenin are the most common alterations associated with tumor development and cancer cell migration or metastasis (9-12) and the presence of mutated $\beta$-catenin is associated with aggressive tumor growth $(9,10)$. In the canonical model of Wnt signaling, $\beta$-catenin activity is regulated by phosphorylation at certain key residues by glycogen synthase kinase-3 $\beta$ (GSK-3 $\beta$ ) and casein kinase $1 \alpha(\mathrm{CK} 1 \alpha)$, which leads to its ubiquitination and subsequent degradation $(9,10)$. Loss of regulation of $\beta$-catenin results in excessive nuclear accumulation and subsequent stimulation of downstream target genes, which includes the genes that encode proteins that play key roles in cell proliferation and tumor growth $(13,14)$. 
Smith et al (15) have reported that UVB radiation induces $\beta$-catenin signaling in keratinocytes. While COX-2 and $\beta$-catenin have been linked to colon cancer, it is unclear whether UVB-induced COX-2-mediated $\beta$-catenin expression is linked in skin cells or whether there is any association between the development of UVB-induced skin tumors and $\beta$-catenin signaling activation. To establish whether these links occur in UVB-exposed skin, we have performed short-term and long-term in vivo animal experiments, in which mice were exposed to acute and chronic UVB exposures and their effect on $\beta$-catenin signaling investigated. We also examined the status of $\beta$-catenin signaling in UVB-induced skin tumors using SKH-1 hairless mice. We show that exposure of mouse to UVB radiation induced dose- and time-dependent activation of $\beta$-catenin in the skin, and that this effect of UVB radiation on $\beta$-catenin activation was dependent on UVB-induced inflammatory mediators or $\mathrm{COX}-2 / \mathrm{PGE}_{2}$ expression. To verify this link, we also used COX-2-deficient mice and a high-fat diet (HF-diet)-induced inflammation-mediated mouse skin tumor model.

\section{Materials and methods}

Animals. The 6- to 7-week-old female $\mathrm{C} 3 \mathrm{H} / \mathrm{HeN}$ and SKH-1 hairless mice used in this study were purchased from Charles River Laboratory (Wilmington, MA). The breeding pairs of COX-2-deficient mice were kindly provided by Dr R. Langenbach of National Institutes of Environmental Health Sciences (NIEHS/NIH), and a colony of COX-2deficient mice was maintained in our animal resource facility, as described (16). COX-2-deficient mice are heterozygous and can survive for several weeks and lack of any clear phenotypical differences with their wild-types. Homozygous mice are resistant in breeding. All mice were maintained under standard housing conditions of a 12-h dark/12-h light cycle, relative humidity of $50 \pm 10 \%$ and a temperature of $24 \pm 2^{\circ} \mathrm{C}$. The animal protocol used in this study was approved by the Institutional Animal Care and Use Committee of the University of Alabama at Birmingham.

Reagents and antibodies. Antibodies specific for COX-2, EP2, EP4, proliferating cell nuclear antigen (PCNA), $\beta$-catenin (specific for immunostaining) and associated secondary antibodies (Alexafluor 488-conjugated) were purchased from Santa Cruz Biotechnology (Santa Cruz, CA). The $\mathrm{PGE}_{2}$ immunoassay kit, indomethacin and AH6809 were purchased from Cayman Chemical (Ann Arbor, MI). The antibodies for $\beta$-catenin, cyclin D1, cyclin D2, CK1 $\alpha$, GSK-3 $\beta$, matrix metalloproteinase (MMP)-2, MMP-9 and c-Myc were obtained from Cell Signaling Technology (Beverly, MA).

UVB irradiation of mice. The dorsal hair of $\mathrm{C} 3 \mathrm{H} / \mathrm{HeN}$ and COX-2-deficient mice was shaved with electric clippers at least $24 \mathrm{~h}$ before UVB exposure. The backs of the mice were UVB irradiated as described earlier (6) using a band of four FS20 UVB lamps (Daavlin, UVA/UVB Research Irradiation Unit, Bryan, $\mathrm{OH}$ ) equipped with an electronic controller to regulate UV dosage. The UV lamps emit UVB (280-320 nm; $\sim 80 \%$ of total energy) and UVA $(320-375 \mathrm{~nm} ; \sim 20 \%$ of total energy). The peak emission of UV radiation is at $314 \mathrm{~nm}$. This UV unit enables us to enter the UV dose in millijoules and variations in energy output are compensated automatically so that the desired UV dose is delivered.

Treatment of mice with indomethacin and EP2 receptor antagonist. To assess the effect of COX-2 inhibitor (indomethacin) or EP2 antagonist (AH6809) on UVB-induced inflammatory responses and the expression levels of $\beta$-catenin proteins, the mice were treated topically with indomethacin (50 $\mu \mathrm{g}$ in $0.2 \mathrm{ml}$ acetone) $(17,18)$ or AH6809 $(25 \mu \mathrm{g}$ in $0.2 \mathrm{ml}$ acetone) $(17,19)$, which were applied to the shaved dorsal skin of $\mathrm{C} 3 \mathrm{H} / \mathrm{HeN}$ mice $30 \mathrm{~min}$ before UVB exposure. Control animals were treated topically with acetone $(0.2 \mathrm{ml}$ acetone). Animals were sacrificed $24 \mathrm{~h}$ after the last UVB exposure, and skin samples were collected for the analysis of protein biomarkers. Each group had 4-5 animals.

Immunoassay analysis of $P G E_{2}$. Skin samples were homogenized in $100 \mathrm{mM}$ phosphate buffer, $\mathrm{pH} 7.4$, as described earlier using a polytron homogenizer (PT3100, Fisher Scientific, Rockford, IL). The supernatants were collected after centrifugation for analysis of the levels of $\mathrm{PGE}_{2}$ using the Cayman $\mathrm{PGE}_{2}$ Enzyme Immunoassay Kit following the manufacturer's protocol (Cayman Chemical).

Western blot analysis. The skin or skin tumor samples were pooled from two or three mice in each group $(n=4-5$ per group), and at least 2 sets of samples were prepared for western blot analysis. Skin or tumor lysates were prepared for western blot analysis as described previously (6). Proteins (30-60 $\mu \mathrm{g})$ were resolved by $8-10 \%$ sodium dodecyl sulfate/polyacrylamide gel electrophoresis and transferred onto nitrocellulose membranes. The membranes were incubated in blocking buffer for $1 \mathrm{~h}$ at room temperature and then incubated with the primary antibodies overnight at $4^{\circ} \mathrm{C}$. The membrane was then washed with phosphate buffered saline (PBS) and incubated with HRP-conjugated secondary antibody. Protein bands were visualized using the enhanced chemiluminescence detection reagents. Equal loading of proteins on the gel was verified by re-probing the membrane with anti- $\beta$-actin antibody.

Immunofluorescent detection of $\beta$-catenin. Immunohistochemical detection of $\beta$-catenin in skin tumor samples was performed as detailed previously (11). Briefly, sections were deparaffinized and rehydrated in a graded series of alcohols. Following the antigen retrieval process, cells were permeabilized with $0.2 \%$ Triton X-100 (Sigma, St. Louis, MO) in PBS and then incubated with $\beta$-catenin-specific antibody for $2 \mathrm{~h}$ at room temperature. The cells were washed with PBS buffer and $\beta$-catenin was detected by an Alexafluor 488-conjugated secondary antibody. Sections were mounted with Vectashield mounting medium for fluorescence and stained with DAPI (Vector Laboratories, Burlingame, CA). Sections were observed using a fluorescence detection-equipped microscope and photographed.

Statistical analysis. The statistical significance of difference for $\mathrm{PGE}_{2}$ between the values of control and treatment groups was determined using analysis of variance (ANOVA). $\mathrm{P}<0.05$ was considered to indicate a statistically significant difference. 
A Time-dependent

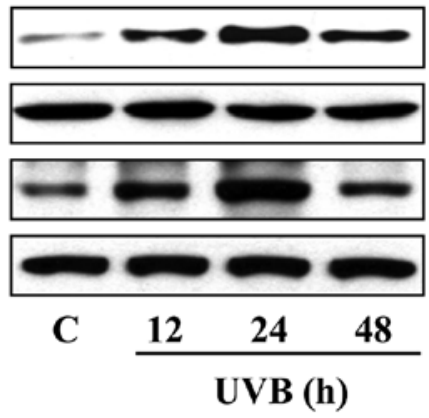

B

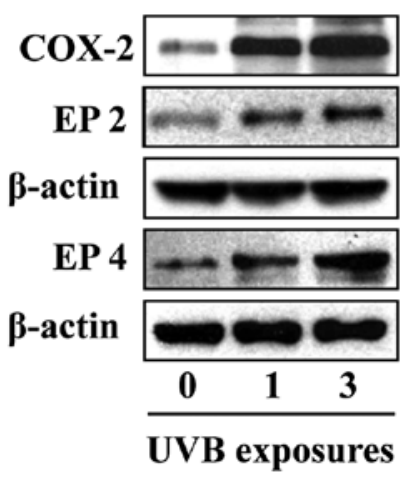

UVB dose-dependent

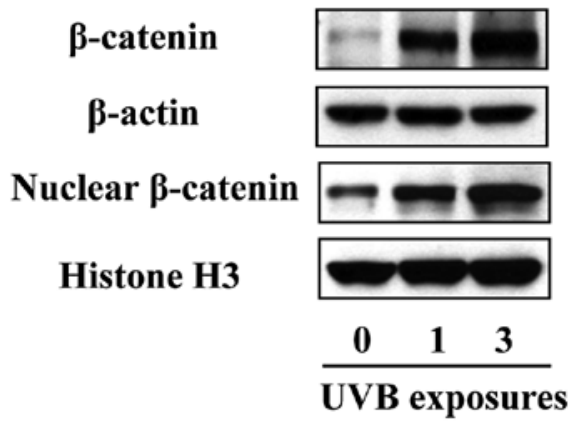

C

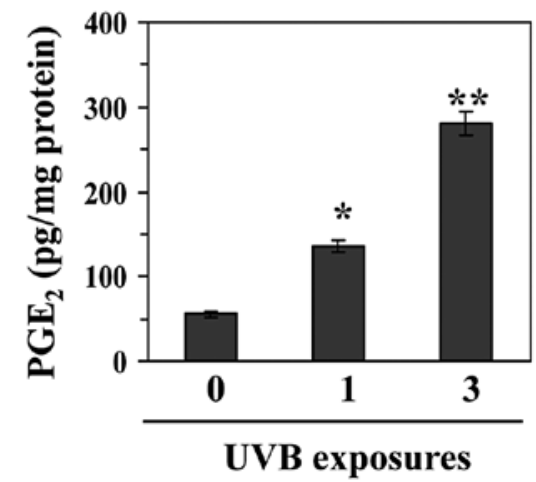

Figure 1. Detection of $\beta$-catenin expression in UVB-exposed mouse skin. $\mathrm{C} 3 \mathrm{H} / \mathrm{HeN}$ mice were subjected to UVB irradiation $\left(180 \mathrm{~mJ} / \mathrm{cm}^{2}\right)$, and euthanized at the indicated time points after UVB irradiation, $n=4-5 / g r o u p$. Skin lysates were prepared and subjected to western blot analysis. (A) Time- and dose-dependent effect of UVB exposure on the expression levels of $\beta$-catenin. (B) Effect of single vs. multiple UVB exposures on COX-2 expression and the expression of PGE 2 receptors in mouse skin. Mice were exposed to UVB radiation $\left(180 \mathrm{~mJ} / \mathrm{cm}^{2}\right)$ once or three times and euthanized $24 \mathrm{~h}$ after the last UVB exposure. Skin lysates were prepared and subjected to western blot analysis. (C) Effect of UVB irradiation on PGE $_{2}$ production. The results are expressed in terms of pg/mg protein. Significant difference vs control, ${ }^{*} \mathrm{P}<0.05,{ }^{* *} \mathrm{P}<0.001$.

\section{Results}

UVB irradiation enhances the levels of $\beta$-catenin in mouse skin. $\mathrm{C} 3 \mathrm{H} / \mathrm{HeN}$ mice were exposed to a single dose of UVB $\left(180 \mathrm{~mJ} / \mathrm{cm}^{2}\right)$, and mice were sacrificed at different time points after UVB irradiation to determine the kinetics of UVB-induced $\beta$-catenin expression. Skin samples were collected and lysates were subjected to western blot analysis to determine the levels of $\beta$-catenin. As shown in Fig. 1A (left panel), the expression of $\beta$-catenin in the mouse skin was upregulated at 12 and $24 \mathrm{~h}$ after UVB exposure and then declined at or after $48 \mathrm{~h}$ compared to the expression level of $\beta$-catenin at $24 \mathrm{~h}$ after UV irradiation. The levels of nuclear $\beta$-catenin followed the same time course. We also compared the effect of single and multiple (3X) exposures of UVB irradiation on the expression of $\beta$-catenin in the skin. As shown in Fig. 1A (right panel), the effects of UVB radiation on the levels of cytosolic and nuclear $\beta$-catenin expression were exacerbated when the skin was exposed to UVB radiation three times on three consecutive days as compared with the levels of $\beta$-catenin in the skin after a single UVB exposure.

$U V B$-induced activation of $\beta$-catenin is associated with enhanced expression of $C O X-2, P G E_{2}$ and $P G E_{2}$ receptors. To determine whether induction of $\beta$-catenin after UVB irradiation is linked with UVB-induced inflammation and inflammatory mediators, we analyzed the levels of COX-2, $\mathrm{PGE}_{2}$ and the receptors of $\mathrm{PGE}_{2}$ (EP2 and EP4) in the mouse skin. For this purpose mice were exposed either once or three times on three consecutive days. Skin samples were collected $24 \mathrm{~h}$ after the last UVB exposure. Western blot analysis revealed that exposure of the skin to UVB resulted in higher expression of COX-2 (Fig. 1B) and $\mathrm{PGE}_{2}$ (Fig. 1C) as compared to non-UVB-exposed mouse skin and this higher COX-2 and $\mathrm{PGE}_{2}$ expression was dependent on the number of UVB exposures. As most of the biological functions of $\mathrm{PGE}_{2}$ are mediated through its receptors, we examined the levels of two $\mathrm{PGE}_{2}$ receptors, EP2 and EP4, which are the more prominent receptors in the skin. As shown in Fig. 1B, the levels of EP2 and EP4 were higher in UVB-irradiated skin compared to non-UVB-exposed skin and the levels of these receptors were higher in skin which had been irradiated three times with UVB as compared with skin that had been subject to only one UVB exposure.

COX-2-deficient mice are resistant to upregulation of $\beta$-catenin in UVB-exposed skin. If COX-2 stimulates UVB-induced expression of $\beta$-catenin in mice, then COX-2-deficiency in mice should block or inhibit UVB-induced upregulation of $\beta$-catenin. Thus, to confirm the role of COX-2 or $\mathrm{PGE}_{2}$ in 
COX-2-deficient mice

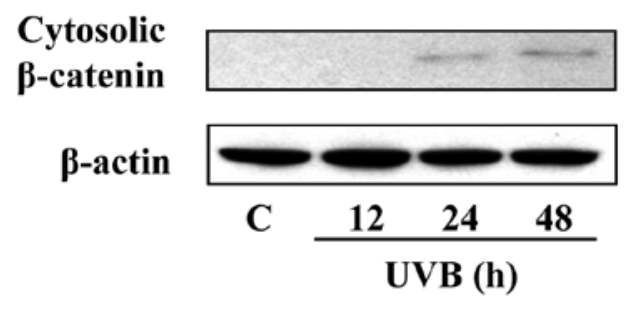

COX-2 wild-type

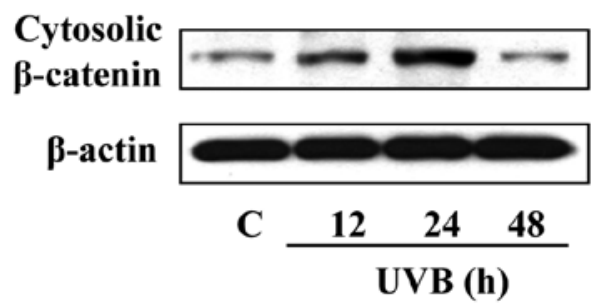

Figure 2. Detection of $\beta$-catenin expression in UVB-exposed mouse skin of COX-2-deficient mice and their wild-type counterparts. Analysis of time-dependent effect of UVB-exposure on the expression of $\beta$-catenin in COX-2-deficient and wild-type mice using western blot analysis.

UVB-induced expression of $\beta$-catenin in mouse skin, we used COX-2-deficient mice $\left(\mathrm{COX}-2^{+/-}\right)$. It is established that these mice do not undergo significant enhancement of UVB-mediated inflammation or inflammatory mediators in the skin, including $\mathrm{PGE}_{2}$. The COX-2-deficient mice were exposed to acute UVB $\left(180 \mathrm{~mJ} / \mathrm{cm}^{2}\right)$ radiation and sacrificed at different time points (12, 24 and 48 h) after UVB exposure. Skin samples were collected and lysates prepared for analysis of $\beta$-catenin by western blot analysis. As shown in Fig. 2, there was no marked increase in the levels of $\beta$-catenin in the UVB-exposed skin of the COX-2-deficient mice at $12 \mathrm{~h}$ after UVB irradiation as compared with non-UVB-exposed skin of COX-2-deficient mice. However, the presence of COX-2 protein was noted at 24 and $48 \mathrm{~h}$ after $\mathrm{UVB}$-irradiation of mice. As the mice used for this study were heterozygous COX-2-deficient, the residual presence of $\beta$-catenin is not surprising. However, the levels of $\beta$-catenin were clearly higher in the UVB-exposed skin of wild-type mice at 12 and $24 \mathrm{~h}$ after UVB exposure. The level of $\beta$-catenin was declined at/or after $48 \mathrm{~h}$ of UVB exposure compared to $24 \mathrm{~h}$ time point. These results indicate that $\mathrm{COX}-2$-deficient mice are resistant to UVB-induced activation of $\beta$-catenin signaling.

Treatment of mice with $C O X-2$ inhibitor or EP2 antagonist inhibits $U V B$-induced activation of $\beta$-catenin signaling in mice. To further identify whether the induction of COX-2 in UVB-exposed skin is responsible for the activation of $\beta$-catenin signaling, the effects of UVB radiation on the activation of $\beta$-catenin was determined in mice that had been administered the COX-2 inhibitor, indomethacin. As most of the biologic functions of $\mathrm{PGE}_{2}$ are mediated through the $\mathrm{PGE}_{2}$ receptor $\mathrm{EP} 2$, we also tested the effects of $\mathrm{PGE}_{2}$ on the activation of $\beta$-catenin signaling by using an EP2 antagonist (AH6809) to block the action of $\mathrm{PGE}_{2}$ in UVB-exposed skin. In these experiments, mice were UVB irradiated on three consecutive days with or without topical application of indomethacin or the AH6809. The mice were sacrificed $24 \mathrm{~h}$ after the last UVB exposure and skin samples processed for protein analysis. As shown in Fig. 3A, western blot analysis data confirmed the levels of cytosolic and nuclear $\beta$-catenin were higher in the UVB-exposed skin than in the non-UVB-irradiated mouse skin. Topical application of indomethacin or EP2 antagonist before each exposure to UVB resulted in lower accumulation of $\beta$-catenin in the mouse skin compared to the levels in the mouse skin that was irradiated to UVB but was not treated with indomethacin or EP2 antagonist. Additionally, we found that the levels of CK1 $\alpha$ and GSK-3 $\beta$
A

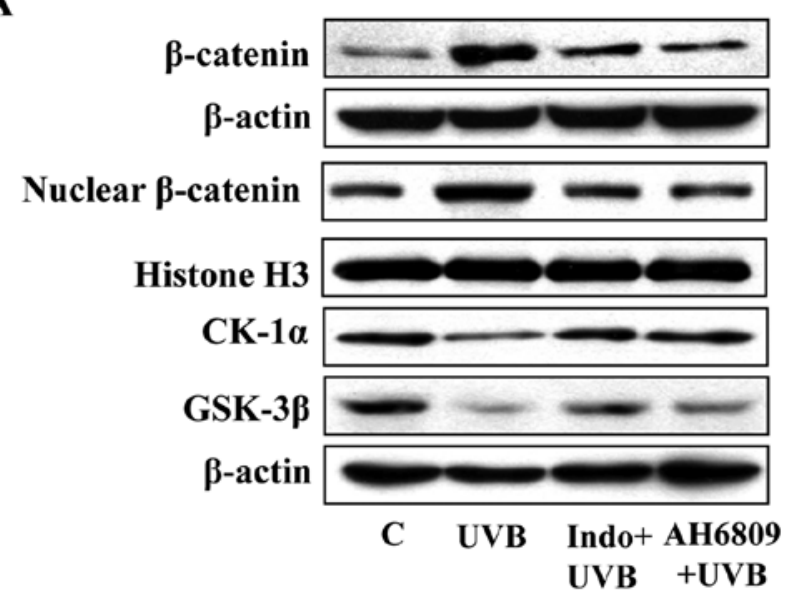

B

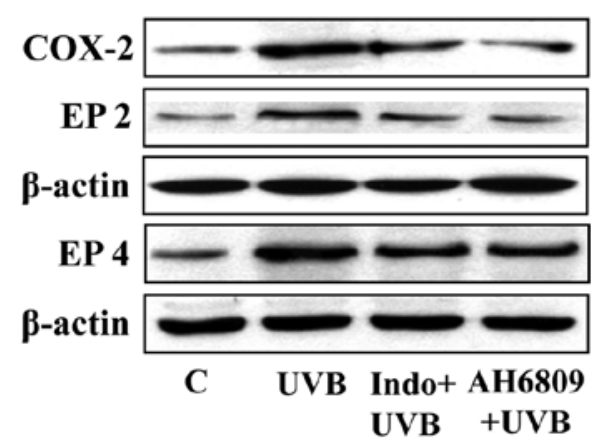

C

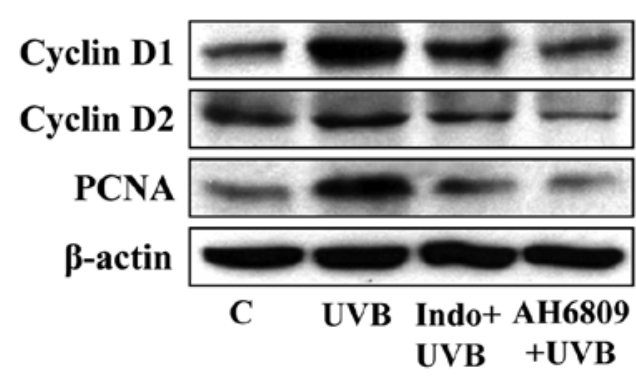

Figure 3. Effect of indomethacin (a COX-2 inhibitor) and AH6809 (an EP2 antagonist) on UVB induced expression of $\beta$-catenin in mouse skin. $\mathrm{C} 3 \mathrm{H} / \mathrm{HeN}$ mice were exposed three times to UVB radiation $\left(180 \mathrm{~mJ} / \mathrm{cm}^{2}\right)$ and euthanized $24 \mathrm{~h}$ after the last UVB exposure. Skin lysates were subjected to western blot analysis, $n=4 /$ group. (A) Treatment of mice with indomethacin or AH6809 reduced UVB induced expressions of $\beta$-catenin and the indicated related signaling proteins, (B) inhibited the levels of COX-2, EP2 and EP4 receptors, and $(\mathrm{C})$ inhibited the levels of cell proliferation biomarkers in mouse skin. Equal loading of proteins on the gels was verified using anti- $\beta$-actin antibody. 
$\mathbf{A}$

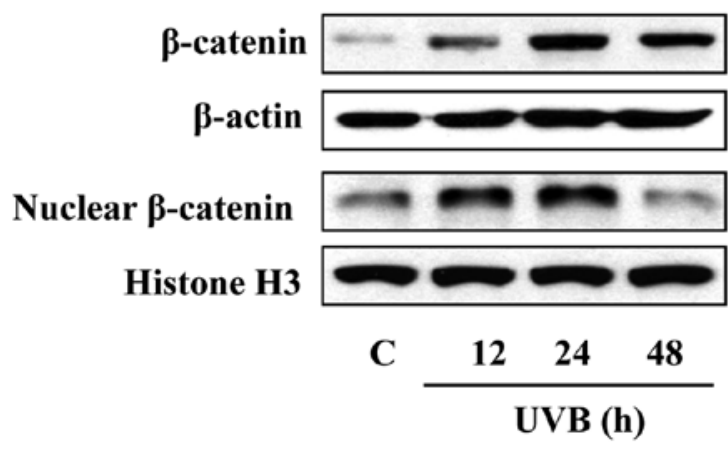

C

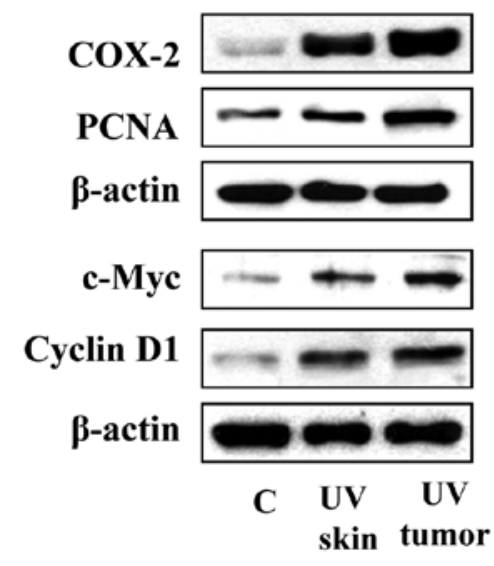

B

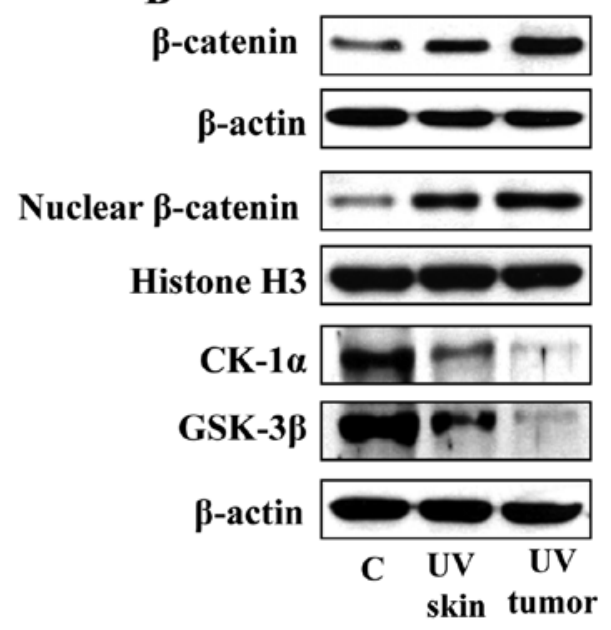

Figure 4. Effect of UVB irradiation on $\beta$-catenin in SKH-1 hairless mouse skin and skin tumors. (A) Time-dependent effect of UVB radiation on $\beta$-catenin in SKH-1 hairless mouse skin. SKH-1 hairless mice were exposed to UVB radiation $\left(180 \mathrm{~mJ} / \mathrm{cm}^{2}\right)$ and euthanized at different time points. Skin lysates were subjected to western blot analysis. (B) SKH-1 hairless mice were subjected to a photocarcinogenesis protocol in which mice were exposed to UVB radiation $\left(180 \mathrm{~mJ} / \mathrm{cm}^{2}\right)$ three times a week for 24 weeks. At the end of experiment, tumors and tumor-uninvolved skin samples were harvested and lysates were prepared for western blot analysis. The effects of UVB radiation on $\beta$-catenin and the indicated signaling proteins were determined. (C) The levels of COX-2 and downstream protein targets of $\beta$-catenin in UVB-exposed skin and UVB-induced skin tumors were determined using western blot analysis.

were elevated in the skin of mice which were UVB-exposed and were treated with either indomethacin or EP2 antagonist compared to the UVB-irradiated skin of mice which were not treated with indomethacin or EP2 antagonist.

Effect of indomethacin and EP2 antagonist on UVB-induced inflammatory responses. As overexpression of COX-2 and $\mathrm{PGE}_{2}$ in UVB-exposed skin are prominent biomarkers of inflammation and have a role in activation of $\beta$-catenin signaling, we determined the effects of indomethacin and the EP2 antagonist on UVB-induced expression of COX-2 in parallel to the analysis of the effects on the expression of $\beta$-catenin. As shown in Fig. 3B, the results confirmed that the levels of COX-2, EP2 and EP4 were higher in the UVB-exposed skin of mice than the non-UVB-exposed mouse skin. As would be anticipated, the levels of COX-2 were markedly lower in the indomethacin-treated UVB-exposed skin than the UVB-exposed untreated mouse skin and there was a similar reduction in the levels of EP2 and EP4. Similar effects on $\mathrm{COX}-2, \mathrm{EP} 2$ and $\mathrm{EP} 4$ proteins were found when the mouse skin was treated with EP2 antagonist prior to UVB exposure (Fig. 3B). In the same set of experiments, we determined the effects of indomethacin and EP2 antagonist on biomarkers of proliferation, including PCNA, cyclin D1 and cyclin D2. As shown in Fig. 3C, treatment of mice with indomethacin or EP2 antagonist inhibited UVB-enhanced expression of PCNA, cyclin D1 and cyclin D2 as compared to the expression of these biomarkers of proliferation in the skin of UVB-irradiated mice which were not treated with indomethacin or EP2 antagonist.

Analysis of UVB-induced activation of $\beta$-catenin signaling in the SKH-1 hairless mouse model. To further verify our results obtained in $\mathrm{C} 3 \mathrm{H} / \mathrm{HeN}$ mouse model with respect to UVB-induced $\beta$-catenin, we conducted experiments using the SKH-1 hairless mouse model. In this mouse model, the animals were exposed to acute UVB irradiation $\left(180 \mathrm{~mJ} / \mathrm{cm}^{2}\right)$ and sacrificed at 12, 24 and $48 \mathrm{~h}$ after UVB irradiation. Skin lysates were subjected to the analysis of the levels of $\beta$-catenin using western blot analysis. As shown in Fig. 4A, the levels of $\beta$-catenin and nuclear $\beta$-catenin were upregulated at the 12 and $24 \mathrm{~h}$ time points and thereafter the levels declined, indicating a time course response similar to that observed in the $\mathrm{C} 3 \mathrm{H} / \mathrm{HeN}$ mice (Fig. 1A). The SKH-1 hairless mouse is a well-established model for analysis of UVB-induced skin tumor formation. We therefore exposed the SKH-1 hairless mice to UVB radiation 3 times per week for total of 24 weeks to determine the long-term effects of UV radiation on $\beta$-catenin signaling and its association with tumor formation. After 24 weeks of UVB irradiation, mice were sacrificed and tumors and tumor uninvolved skin samples collected for the analysis of $\beta$-catenin-associated signaling proteins. As shown in Fig. 4B, western blot analysis revealed that long-term exposure of the mice to UVB irradiation resulted in upregulation of the levels of $\beta$-catenin compared with the skin samples obtained from the non-UVB-irradiated control mice. The levels of $\beta$-catenin in tumor samples were higher than the UVB-irradiated skin samples obtained from the mice in the same group. Additionally, the levels of CK1 $\alpha$ and GSK-3 $\beta$ were reduced in UVB-exposed skin as well as in tumor samples as compared to non-UVB-exposed control skin. 
A

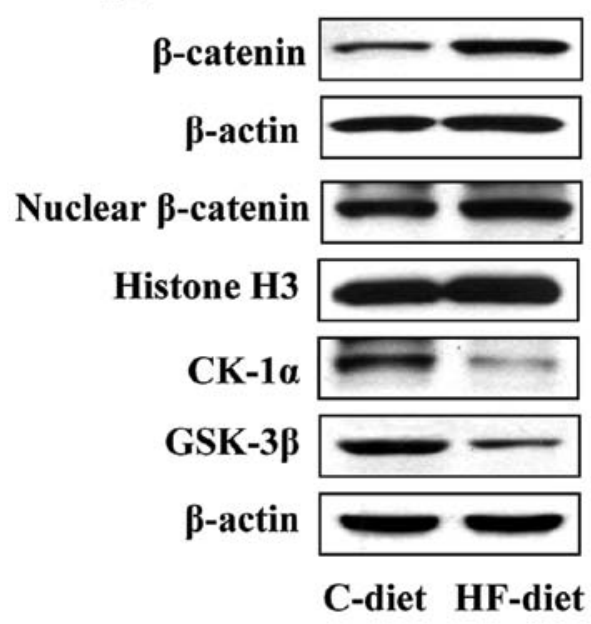

B Immunofluorescence staining of $\beta$-catenin

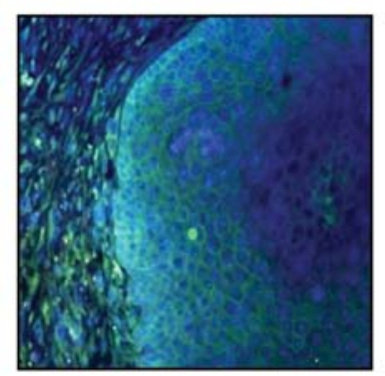

C-diet

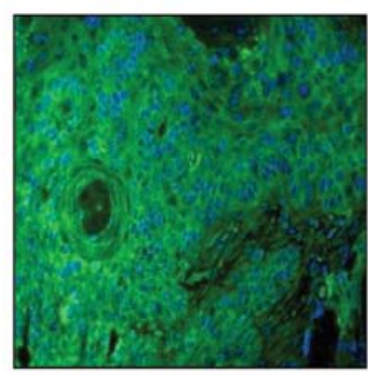

HF-diet
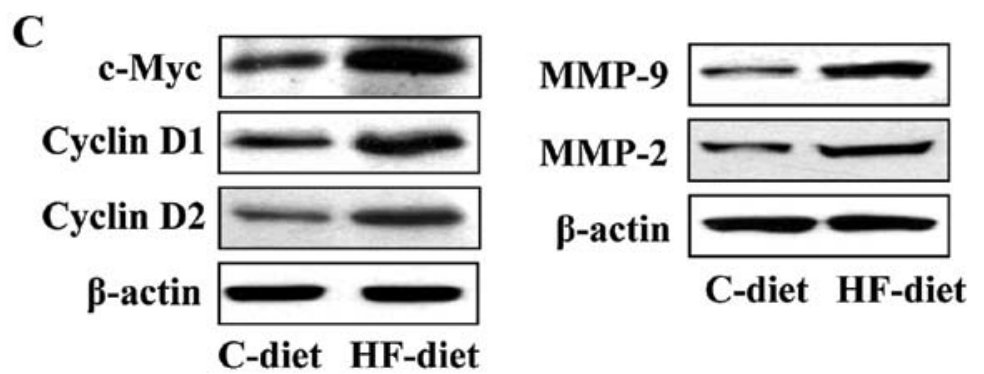

Figure 5. Effect of HF-diet-induced inflammation on the levels of $\beta$-catenin in UVB-induced skin tumors in SKH-1 hairless mice. Mice were given either the HF-supplemented AIN76A diet (HF-diet) or the unsupplemented control AIN76A diet (C-diet) and subjected to a standard photocarcinogenesis protocol for 24 weeks. At the termination of the experiment, mice were sacrificed, the tumors harvested and tumor lysates subjected to western blot analysis of $\beta$-catenin and the indicated related molecular targets. (A) Effect of HF-diet-induced inflammation on $\beta$-catenin signaling proteins in UVB-induced skin tumors. (B) Immunohistochemical detection of $\beta$-catenin in skin tumors of HF-diet-fed and C-diet-fed mice. $\beta$-catenin-positive cells appear green. (C) Western blot analyses of proteins of downstream targets of $\beta$-catenin in skin tumors, $n=4$. Equal loading of proteins on the gels is shown by probing the stripped membrane with anti- $\beta$-actin antibody.

To further verify the potential link between the expression of UVB-induced $\beta$-catenin and the expression of biomarkers of UVB-induced inflammation/proliferation, the skin and tumor samples from the same experiment were analyzed for expression of various biomarkers of these responses. The results of western blot analysis revealed that the expression levels of COX-2, PCNA, c-Myc and cyclin D1 were higher in UVB-induced skin tumors and UVB-exposed skin (tumor uninvolved) as compared with the skin of non-UVB-exposed control animals (Fig. 4C).

Status of $\beta$-catenin and $\beta$-catenin signaling molecules in high-fat diet-induced skin tumors in UVB-irradiated skin. In our recent studies, we found that administration of the HF-diet enhances UVB-induced skin carcinogenesis in SKH-1 hairless mice in terms of tumor multiplicity and tumor growth/size compared to the skin carcinogenesis in control-diet-fed mice (20). In these experiments, we also found that the enhancement of photocarcinogenesis observed on administration of the HF-diet to mice was associated with the higher levels of inflammation and inflammatory mediators than were observed in skin of the mice fed a control diet. Based on these observations, we used this tumor model in our present study to determine whether HF-diet-induced inflammation in UVB-induced skin tumor is associated with enhancement in the levels or activation of $\beta$-catenin. Skin tumor samples from experiments described above were analyzed for biomarkers related to $\beta$-catenin signaling. As shown in Fig. 5A, western blot analysis revealed that the levels of total $\beta$-catenin as well as nuclear $\beta$-catenin were higher in the skin tumor samples from HF-diet-fed mice as compared with the skin tumors of mice fed a control diet. These results were further verified by intracellular staining of $\beta$-catenin in tumor sections. The intensity of intracellular staining of $\beta$-catenin was higher in the skin tumors of HF-diet-fed mice compared with the intracellular staining in the skin tumors of C-diet-fed mice (Fig. 5B). Concomitantly, the levels of CK $1 \alpha$ and GSK-3 $\beta$ were lower in the skin tumors of HF-diet-fed mice than the skin tumors of mice fed the control diet (Fig. 5A). The downstream signaling targets of $\beta$-catenin signaling also were analyzed in the skin tumors from HF-diet and C-diet-fed mice. As shown in Fig. 5C, we found that the levels of c-Myc, cyclin D1, cyclin D2, and MMP-2 and MMP-9 were higher in the skin tumors of HF-diet-fed mice as compared to the skin tumors of mice fed the control diet. These data support our concept that activa- 
tion of $\beta$-catenin signaling in UVB-exposed skin is mediated through enhanced inflammation.

\section{Discussion}

Epidemiological, clinical and experimental studies clearly indicate a link between chronic UVB exposure and greater risk of non-melanoma skin cancer in humans. Although many genetic, epigenetic and environmental factors play a role in the risk of skin cancers, the chronic and sustained generation of inflammation and inflammatory mediators in UVB-exposed skin are considered to be potent regulators of tumor promotion and progression. In particular, UVB-induced overexpression of COX-2 and $\mathrm{PGE}_{2}$ in the skin have been associated with multiple signaling pathways responsible for tumor cell survival, resistance to apoptotic cell death and suppression of immune system $(3,21)$. Despite this, little is known about the potential link between the $\mathrm{COX}-2 / \mathrm{PGE}_{2}$ axis and $\beta$-catenin signaling in UVB-exposed skin and UVB-induced skin tumors. It has been shown that $\mathrm{PGE}_{2}$ can promote the growth of colon cancer cells by activating $\beta$-catenin signaling (8). The study by Smith et al (15) demonstrated that UVB radiation-induced $\beta$-catenin signaling is increased by $\mathrm{COX}-2$ expression in human and mouse keratinocytes, and also in UVB-irradiated mouse skin. Thus, it was interesting to extend the studies on the effect of UVB radiation on $\beta$-catenin signaling and also to check the levels of $\beta$-catenin in UVB-induced skin tumors. In this report, we provide evidence that UVB-induced upregulation of $\mathrm{COX}-2 / \mathrm{PGE}_{2}$ results in activation of $\beta$-catenin signaling in the mouse skin and contributes to skin tumor development by using different mouse models.

The data presented herein provide evidence that UVB-induced COX-2 expression and $\mathrm{PGE}_{2}$ production promotes $\beta$-catenin signaling in UVB-exposed mouse skin. The lack of upregulation and activation of $\beta$-catenin signaling in UVB-exposed COX-2-deficient mice supports the evidence that UVB-induced COX-2 overexpression may be necessary for the activation of $\beta$-catenin. The activation of $\beta$-catenin signaling in UVB-exposed skin under the influence of inflammatory mediators, such as $\mathrm{COX}-2$ and $\mathrm{PGE}_{2}$, was verified using two strains of mice, $\mathrm{C} 3 \mathrm{H} / \mathrm{HeN}$ and $\mathrm{SKH}-1$ hairless mice (Figs. 1 and 4). Additionally, we found that treatment of mice with indomethacin (an inhibitor of COX-2) and the EP2 antagonist suppressed the accumulation as well as activation of $\beta$-catenin signaling in UVB-exposed skin, and established their effects on CK1 $\alpha$, GSK-3 $\beta$ and downstream targets of cell proliferation (Fig. 3).

As $\beta$-catenin has been shown to have a role in tumor growth (8), we subjected SKH-1 hairless mice to a standard photocarcinogenesis protocol $(20,22)$. At the termination of the photocarcinogenesis experiment, mice were sacrificed and UVB-induced skin tumors and tumor uninvolved skin samples were collected and analyzed for the biomarkers of inflammation and the molecules of $\beta$-catenin signaling. The levels of $\beta$-catenin were markedly higher in chronically UVB-exposed skin and UVB-induced skin tumors compared with the skin samples from non-UVB-exposed control mice. The levels of COX-2 expression and downstream targets of $\beta$-catenin signaling were also upregulated in skin tumors suggesting the role of
COX-2 overexpression in activation of $\beta$-catenin signaling in UVB-induced skin tumors (Fig. 4).

Finally, to further support the evidence that UVB-induced inflammation, specifically overexpression of COX-2 and $\mathrm{PGE}_{2}$, has a role in the activation of $\beta$-catenin signaling, we used a high fat-diet-induced inflammation model (20). We have found that administration of high-fat diet induces higher expression of COX-2 and $\mathrm{PGE}_{2}$ in UVB-induced skin tumors than the skin tumors in control-diet-fed mice (20). We used this tumor model, and determined the relationship between $\mathrm{COX}-2 / \mathrm{PGE}_{2}$ and activation of $\beta$-catenin signaling. As shown in Fig. 5, we found that the levels of $\beta$-catenin and their downstream targets, such as c-Myc, cyclin D1, cyclin D2, MMP-2 and MMP-9, were higher in skin tumors of high-fat diet-fed mice compared with the skin tumors of control-diet fed mice. These data provide additional evidence that overexpression of $\mathrm{COX}-2$ and $\mathrm{PGE}_{2}$ have a role in activation of $\beta$-catenin signaling in UVB-exposed skin and UVB-induced skin tumorigenesis. In addition to $\mathrm{COX}-2 / \mathrm{PGE}_{2}$, UVB-induced expression of epidermal growth factor receptor (EGFR) and Akt also has been shown to be involved in activation of $\beta$-catenin signaling. EGFR activation promotes $\beta$-catenin signaling by promoting disassociation of $\beta$-catenin from $\alpha$-catenin (23). There is evidence that $\beta$-catenin signaling contributes to chemically-induced skin cancer. In the two-stage chemical skin carcinogenesis in mice, an increased accumulation of nuclear $\beta$-catenin was observed (24). These findings in combination with our data suggest that UVB-induced $\beta$-catenin signaling contributes to skin carcinogenesis and that it is stimulated by UVB-induced inflammation. Thus, this study suggests that UVB-induced skin cancer can be inhibited by targeting $\beta$-catenin signaling.

\section{Acknowledgements}

Authors thank Dr Fiona Hunter for editorial assistance.

\section{References}

1. Housman TS, Feldman SR, Williford PM, Fleischer AB Jr, Goldman ND, Acostamadiedo JM and Chen GJ: Skin cancer is among the most costly of all cancers to treat for the medicare population. J Am Acad Dermatol 48: 425-429, 2003.

2. American Cancer Society. Cancer Facts and Figures, 2010. American Cancer Society, Atlanta, GA, 2010.

3. Mukhtar H and Elmets CA: Photocarcinogenesis: Mechanisms, models and human health implications. Photochem Photobiol 63: 355-447, 1996

4. Katiyar SK: Oxidative stress and photocarcinogenesis: Strategies for prevention. In: Oxidative Stress, Disease and Cancer. Singh KK (ed). Imperial College Press, London, pp933-964, 2006.

5. Rivas JM and Ullrich SE: The role of IL-4, IL-10, and TNF-alpha in the immune suppression induced by ultraviolet radiation. J Leukoc Biol 56: 769-775, 1994.

6. Meeran SM, Akhtar S and Katiyar SK: Inhibition of UVB-induced skin tumor development by drinking green tea polyphenols is mediated through DNA repair and subsequent inhibition of inflammation. J Invest Dermatol 129: 1258-1270, 2009.

7. Katiyar SK, Matsui MS and Mukhtar H: Kinetics of UV light-induced cyclobutane pyrimidine dimers in human skin in vivo: An immunohistochemical analysis of both epidermis and dermis. Photochem Photobiol 72: 788-793, 2000

8. Castellone MD, Teramoto H, Williams BO, Druey KM and Gutkind JS: Prostaglandin E2 promotes colon cancer cell growth through a Gs-axin-beta-catenin signaling axis. Science 310: 1504-1510, 2005. 
9. Gavert $\mathrm{N}$ and Ben-Ze'ev A: $\beta$-catenin signaling in biological control and cancer. J Cell Biochem 102: 820-828, 2007.

10. Klaus A and Birchmeier W: Wnt signalling and its impact on development and cancer. Nat Rev Cancer 8: 387-398, 2008.

11. Vaid M, Prasad R, Sun Q and Katiyar SK: Silymarin targets $\beta$-catenin signaling in blocking migration/invasion of human melanoma cells. PLoS One 6: e23000, 2011.

12. Rimm DL, Caca K, Hu G, Harrison FB and Fearon ER: Frequent nuclear/cytoplasmic localization of beta-catenin without exon 3 mutations in malignant melanoma. Am J Pathol 154: 325-329, 1999.

13. Li YJ, Wei ZM, Meng YX and Ji XR: Beta-catenin up-regulates the expression of cyclinD1, c-myc and MMP-7 in human pancreatic cancer: relationships with carcinogenesis and metastasis. World J Gastroenterol 11: 2117-2123, 2005.

14. Lowy AM, Clements WM, Bishop J, Kong L, Bonney T, Sisco K, Aronow B, Fenoglio-Preiser C and Groden J: $\beta$-catenin/Wnt signaling regulates expression of the membrane type 3 matrix metalloproteinase in gastric cancer. Cancer Res 66: 4734-4741, 2006.

15. Smith KA, Tong X, Abu-Yousif AO, Mikulec CC, Gottardi CJ, Fischer SM and Pelling JC: UVB radiation-induced $\beta$-catenin signaling is enhanced by COX-2 expression in keratinocytes. Mol Carcinog 51: 734-745, 2012

16. Langenbach $\mathrm{R}$, Loftin $\mathrm{C}$, Lee $\mathrm{C}$ and Tiano $\mathrm{H}$ : Cyclooxygenase knockout mice: models for elucidating isoform-specific functions. Biochem Pharmacol 58: 1237-1246, 1999.

17. Chun KS and Langenbach R: The prostaglandin E2 receptor, EP2, regulates survivin expression via an EGFR/STAT3 pathway in UVB-exposed mouse skin. Mol Carcinog 50: 439-448, 2011.
18. Chun KS, Akunda JK and Langenbach R: Cyclooxygenase-2 inhibits UVB-induced apoptosis in mouse skin by activating the prostaglandin E2 receptors, EP2 and EP4. Cancer Res 67: 2015-2021, 2007.

19. Chun KS, Lao HC, Trempus CS, Okada M and Langenbach R: The prostaglandin receptor EP2 activates multiple signaling pathways and beta-arrestin 1 complex formation during mouse skin papilloma development. Carcinogenesis 30: 1620-1627, 2009.

20. Vaid M, Singh T, Prasad R and Katiyar SK: Intake of high-fat diet stimulates the risk of ultraviolet radiation-induced skin tumors and malignant progression of papillomas to carcinoma in SKH-1 hairless mice. Toxicol Appl Pharmacol 274: 147-155, 2013.

21. Prasad R and Katiyar SK: Prostaglandin E2 promotes ultraviolet radiation-induced immune suppression through DNA hypermethylation. Neoplasia 15: 795-804, 2013.

22. Vaid M, Sharma SD and Katiyar SK: Honokiol, a phytochemical from the Magnolia plant, inhibits photocarcinogenesis by targeting UVB-induced inflammatory mediators and cell cycle regulators: Development of topical formulation. Carcinogenesis 31: 2004-2011, 2010.

23. Ji H, Wang J, Nika H, Hawke D, Keezer S, Ge Q, Fang B, Fang $X$, Fang D, Litchfield DW, Aldape K and Lu Z: EGF-induced ERK activation promotes CK2-mediated disassociation of alpha-catenin from beta-catenin and transactivation of beta-catenin. Mol Cell 36: 547-559, 2009.

24. Bhatia N and Spiegelman VS: Activation of Wnt/beta-catenin/Tcf signaling in mouse skin carcinogenesis. Mol Carcinog 42: 213-221, 2005. 